\title{
CONFIGURACIONES COGNITIVAS Y NIVELES DE ALGEBRIZACIÓN DE UN GRUPO DE ESTUDIANTES UNIVERSITARIOS DE LA FCEQYN
}

\author{
Silvia Caronía* \\ Universidad Nacional de Misiones, Argentina \\ silvca2@gmail.com \\ Norma Martyniuk** \\ Universidad Nacional de Misiones, Argentina \\ norma_martyniuk@yahoo.com.ar
}

Recibido: 16/08/2019 Aceptado: 11/12/2019

\section{Resumen}

En éste trabajo y con los aportes de la Teoría del Enfoque Ontosemiótico (EOS) como herramienta de análisis, se pretende examinar a través de la resolución de un problema, las configuraciones cognitivas y niveles de algebrización, teniendo en cuenta las características de los razonamientos algebraicos puestos en juego, las prácticas matemáticas realizadas y las dificultades que presentaron un grupo de estudiantes del profesorado en matemática de la Facultad de Ciencias Exactas, Químicas y Naturales de la Universidad Nacional de Misiones, Argentina durante los años 2016-2018.

Palabras clave: Práctica matemática - Niveles de algebrización - Razonamiento algebraico.

\begin{abstract}
In this work and with contributions of Onto Semiotic Approach (OSA) as analysis tool, examination problem is meant through cognitive configurations and algebrization levels, taking into account algebraic reasoning at stake, mathematical practices carried out and difficulties faced by a students' group of the Teaching Education College, Misiones National University, Argentina, between 2016-2018.
\end{abstract}

Keywords: Mathematical practice - Levels of algebrization - Algebraic reasoning.

\footnotetext{
* Especialista en Educación Superior, Universidad Nacional de Misiones (UNaM), Argentina. Docente Titular Regular del Profesorado en Matemática de la Facultad de Ciencias Exactas, Químicas y Naturales (FCEQyN-UNaM). Investigadora categoría II del Programa Nacional de Incentivos. Dirige proyectos de investigación en el área educativa. Posee publicaciones a nivel nacional e internacional.

** Profesora en Matemática, Física y Cosmografía de la Facultad de Humanidades y Ciencias Sociales (FHyCS), Universidad Nacional de Misiones (UNaM), Argentina. Docente del Profesorado de Matemática de la Facultad de Ciencias Exactas Químicas y Naturales (FCEQyN), UNaM.
} 


\section{Introducción}

En los últimos años, tanto a nivel nacional como internacional, se viene cuestionando la calidad de los conocimientos que poseen los alumnos de los distintos niveles educativos en el área de matemática, aspecto que se encuentra directamente relacionado con cuestiones inherentes a las dificultades en la enseñanza y el aprendizaje.

La enseñanza y el aprendizaje en el área del álgebra, desde la escolaridad primaria hasta la universidad, continúan siendo motivo de preocupación de numerosos investigadores, que se dedicaron al estudio sobre la iniciación en el trabajo algebraico, además de los problemas y obstáculos que se presentan.

Como formadores de futuros profesores, los docentes del profesorado en Matemática de la UNaM (Universidad Nacional de Misiones, Argentina) vemos con preocupación esta situación, hecho que nos ha llevado, desde hace unos años a realizar investigaciones tendientes a abordar temáticas que tienen como objetivo contribuir en la búsqueda de soluciones a la problemática antes señalada. Investigamos los errores de los alumnos ingresantes a la Universidad, profundizamos sobre temas relacionados con el álgebra que a la luz resultan conflictivos, observamos las instituciones escolares y dentro de ella la práctica del profesor, aspectos todos, que nos han aportado ciertas luces de la enseñanza y el aprendizaje del Álgebra, motivándonos a continuar profundizando en ésta área de la matemática.

Es por todo ello que vimos la necesidad a partir del 2016 de iniciar una investigación poniendo la mirada en nuestros estudiantes del profesorado en matemática, a efectos de identificar cuáles serían sus configuraciones cognitivas, sus niveles de comprensión, porque directa o indirectamente incidirán en el futuro, en la forma que se enfrentarán a sus alumnos. En la medida que desde el profesorado trabajemos éstas cuestiones, podremos de alguna manera, contribuir al cambio en los futuros docentes, como lo manifiestan Godino, Neto, Wilhelmi, Aké, Etchegaray y Lasa (2015): "se precisa que el docente actúe como principal agente de cambio en la introducción y desarrollo del pensamiento algebraico" (Godino, et. al. 2015, p.21).

En esta presentación que es un recorte de un estudio más amplio y con los aportes de la Teoría del Enfoque Ontosemiótico (EOS) como herramienta de análisis, se pretende examinar a través de la resolución de un problema, las configuraciones cognitivas, es decir las características de los razonamientos algebraicos puestos en juego, los tipos de prácticas matemáticas realizadas, como así también los niveles de algebrización y las dificultades que presentaron un grupo de estudiantes.

\section{Metodología y Marco Teórico}

La metodología de investigación utilizada es de tipo cualitativa, caracterizada por el énfasis exploratorio, descriptivo e interpretativo. El marco teórico que guía la misma es la Teoría del Enfoque Ontosemiótico (EOS).

Las principales técnicas efectuadas son la observación y el análisis, utilizando como instrumento una actividad donde se propone una serie de problemas algebraicos, implementadas a un grupo de 60 alumnos (entre 18 y 22 años) del profesorado en Matemática de la Facultad de Ciencias Exactas, Químicas y Naturales de la Universidad Nacional de Misiones (UNaM), Argentina, durante los años 2016 a 2018.

Recolectadas las respuestas de los estudiantes, comenzamos los análisis de la muestra, observando cuáles fueron sus rasgos algebraicos característicos, los razonamientos puestos en juego, los tipos de prácticas matemáticas realizadas, las representaciones del conocimiento, los niveles de algebrización y las dificultades incurridas en la actividad.

En esta presentación uno de los problemas seleccionados, propuestos a los alumnos, ha sido extraído de la tesis doctoral Sadovsky (2003). El mismo involucra las siguientes tareas: producir y establecer la cantidad de soluciones, argumentar sobre el dominio de variación de las variables y encontrar un procedimiento general que permita obtener todas las soluciones. Además, facilita la utilización de las nociones de variable y de dependencia, al introducir un grado de libertad. Cada solución está dada por un par de números y el conjunto solución está constituido por muchos pares. El tener que proponer un procedimiento general para obtener dichas soluciones, permite que se sistematice todos los pares alrededor de una única relación contribuyendo a un proceso de generalización (Sadovsky y Sessa, 2004, p. 5).

En cuanto al Marco Teórico que guía esta investigación, es el Enfoque Ontosemiótico (EOS), el cual uno de los aspectos centrales que se ocupa, es el estudio de las prácticas operativas y discursivas dentro de una actividad matemática. Entendiéndose por práctica matemática a: "toda actuación o expresión (verbal, gráfica, etc.) realizada por alguien para resolver problemas matemáticos, comunicar una solución obtenida, validarla o generalizarla a otros contextos y problemas" (Godino, Batanero 1994, p. 334). 
Estas prácticas emergen con el análisis de los objetos matemáticos primarios y sus procesos en la resolución de un problema. Esta emergencia supone un fenómeno complejo, de ahí que se torna necesario su identificación y estudio conjuntamente con los procesos, a través de un tipo de lenguaje utilizado (gestual, simbólico, verbal o gráfico), un sistema conceptual lógicamente organizado en definiciones, conceptos, procedimientos, proposiciones y argumentos, todos ellos relacionándose entre sí y conformando ciertas configuraciones socio epistémica y cognitivas, dependiendo del sujeto considerado, epistémico (institucional) o personal (Godino, Batanero y Font, 2009, p.4).

En las prácticas algebraicas, Godino et. al. (2015), proponen el análisis de los niveles de algebrización como una posible caracterización del pensamiento algebraico. Al respecto expresan que: "los criterios para delimitar los distintos niveles están basados en el tipo de objetos y procesos matemáticos implicados en la actividad matemática" (Godino et. al., 2015, p.3) y advierten que esos niveles de algebrización se asignan a la actividad realizada por el sujeto y no a las propias tareas, ya que, por más que una tarea esté formulada algebraicamente, la práctica realizada por el alumno podría ser categorizada desde la aritmética.

Los objetos algebraicos que deben ser tenidos en cuenta en una práctica matemática son: a) las relaciones binarias de equivalencia y orden, y sus respectivas propiedades, b) las operaciones y sus propiedades realizadas sobre los elementos de conjuntos diversos, c) las funciones, d) las estructuras, sus tipos y propiedades (Godino, et. al. 2014, p.204).

Estos objetos algebraicos considerados, emergen mediante los respectivos procesos algebraicos. Uno de los procesos algebraicos que se puede presentar es la generalización, como resultado se obtiene un tipo de objeto intensivo, o bien se dice a este proceso, como la generación o inferencia de intensivos. Éste objeto, es la regla que genera los elementos que componen una colección o conjunto finito o infinito. En el sentido inverso al proceso de generalización está el proceso de particularización, cuyo resultado es un objeto extensivo, es decir un objeto particular.

También se pueden exteriorizar otros procesos algebraicos como ser: el de la "unitarización", que es el reconocimiento explícito de intensivos como entidades unitarias. "La formalización y ostensión, nombramiento mediante expresiones simbólico-literales. Transformación, utilización de los objetos intensivos en procesos de cálculo y en nuevas generalizaciones" (Godino et. al., 2014, p.206).

Otras cuestiones que se piensan en el análisis de los niveles de algebrización son los tipos de lenguajes utilizados para enunciar a esos objetos (términos, expresiones, notaciones, gráficos) y el tratamiento que se emplean con esos objetos, por ejemplo: operaciones y sus propiedades, transformaciones, etc. (Aké, Godino y Castro, 2015, p.3)

Teniendo en cuenta lo expresado precedentemente discurrimos que las actividades algebraicas son un tanto complejas en su identificación para el análisis de los razonamientos algebraicos, es por ello que, consideramos para los rasgos característicos, las ideas planteadas por Godino et al. (2015) y clasificados en 6 niveles:

Nivel 0 . Se opera con objetos intensivos de primer grado de generalidad, usando lenguajes natural, numérico, icónico, gestual.

Nivel 1. Se usan objetos intensivos de segundo grado de generalidad, propiedades de la estructura algebraica de $\mathrm{N}$ y la igualdad como equivalencia.

Nivel 2. Se usan representaciones simbólico - literales para referir a los objetos intensivos reconocidos, los cuales están ligados a la información espacial, temporal y contextual; se resuelven ecuaciones de la forma $A x+B=C(A, B, C \in R)$.

Nivel 3. Los símbolos se usan de manera analítica, sin referir a la información contextual. Se realizan operaciones con indeterminadas $O$ variables; se resuelven ecuaciones de la forma $A x+B=C x+D(A, B, C, D \in R)$.

Nivel 4. Se estudian familias de ecuaciones y funciones usando parámetros y coeficientes.

Nivel 5. Se realizan cálculos analíticos (sintácticos) que implican el uso de uno o más parámetros, junto con variables o indeterminadas.

Nivel 6. Se comienza a estudiar estructuras algebraicas en sí mismas, sus definiciones y propiedades estructurales (Godino et. al., 2015, p.19) 


\section{Resultados de los avances}

\section{Configuración Epistémica de Referencia}

Consideramos para nuestro análisis la "Configuración Epistémica de Referencia" que sería tomar el estudio del experto a fin de poder analizar luego las configuraciones cognitivas de los estudiantes (ver Notas bibliográficas 1)

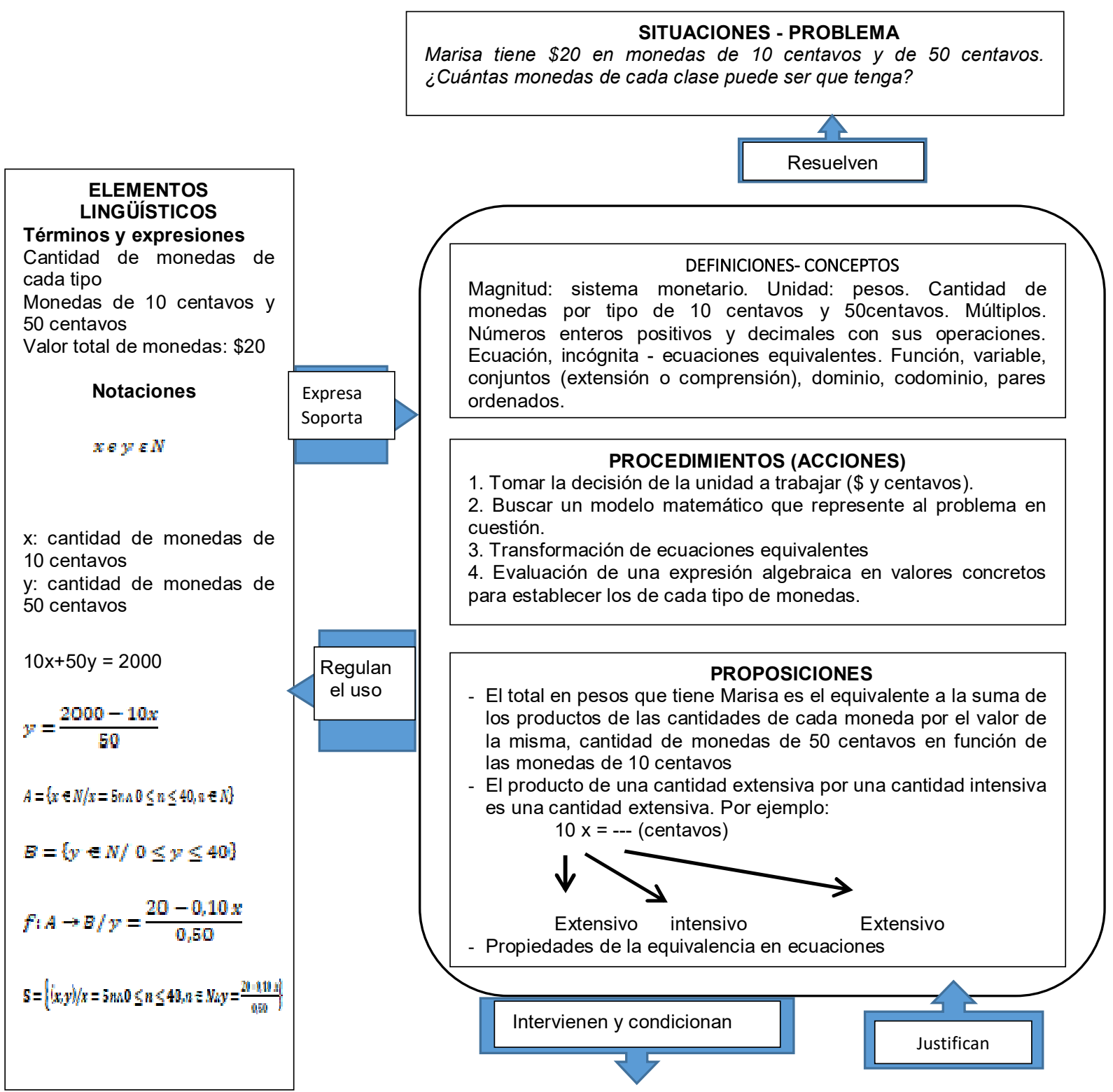




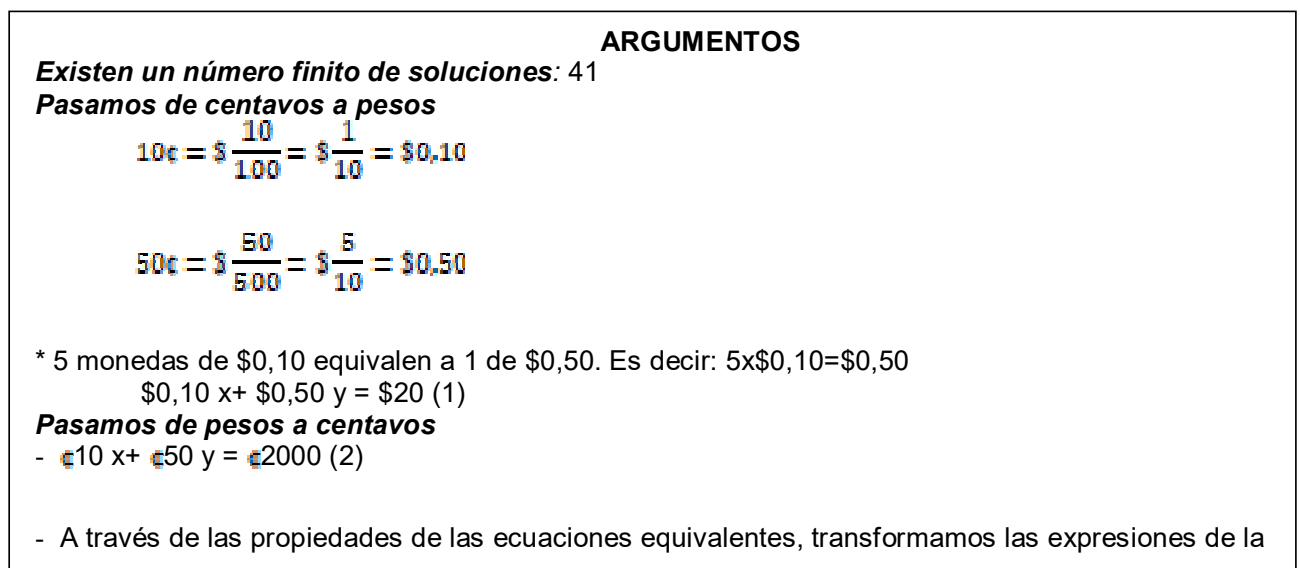

Podemos pensar como una función que va de $A$ en $B$ incluido en el conjunto de los números Naturales:

$$
f\left(A+D / y=\frac{20-0,10 x}{0,50}\right.
$$

Dónde el Dominio y Codominio estarán definidos de la siguiente manera:

$$
A=[x \in N / x=5 n \wedge \leq n \leq 40 n \in N\} ; \quad D=[y \in N / 0 \leq y \leq 40]
$$$$
S=\left\{(x, y) / x=5 m 0 \leq n \leq 40, n \in N A y=\frac{20-0,10 x}{0,50}\right)
$$

Fig.1 Configuración epistémica de referencia

\section{Configuraciones Cognitivas}

Si bien en este trabajo se considera el caso de 5 estudiantes, sus prácticas matemáticas son representativas del total de alumnos analizados. La mirada sobre el quehacer matemático, en la resolución del problema planteado, se realizó cotejando a los mismos con los elementos de la configuración epistémica del experto

\subsection{Configuración Cognitiva del}

\section{Alumno 1}

ELEMENTOS LINGÜÍSTICOS
Términos y expresiones
Cantidad de monedas de cada
tipo
Monedas de 10 centavos y 50
centavos
Valor total de monedas: $\$ 20$
Notaciones
$n:$ monedas de 10 centavos
k: monedas de 50 centavos
$r$ cantidad de monedas de 50
centavos
$200-5 r:$ cantidad de monedas
de 10 centavos
$(200-5 r) n+r k=\$ 20$
$r \varepsilon N, 1 \leq r \leq 39$

\section{SITUACIONES - PROBLEMA}

Marisa tiene $\$ 20$ en monedas de 10 centavos y de 50 centavos. ¿Cuántas monedas de cada clase puede ser que tenga?

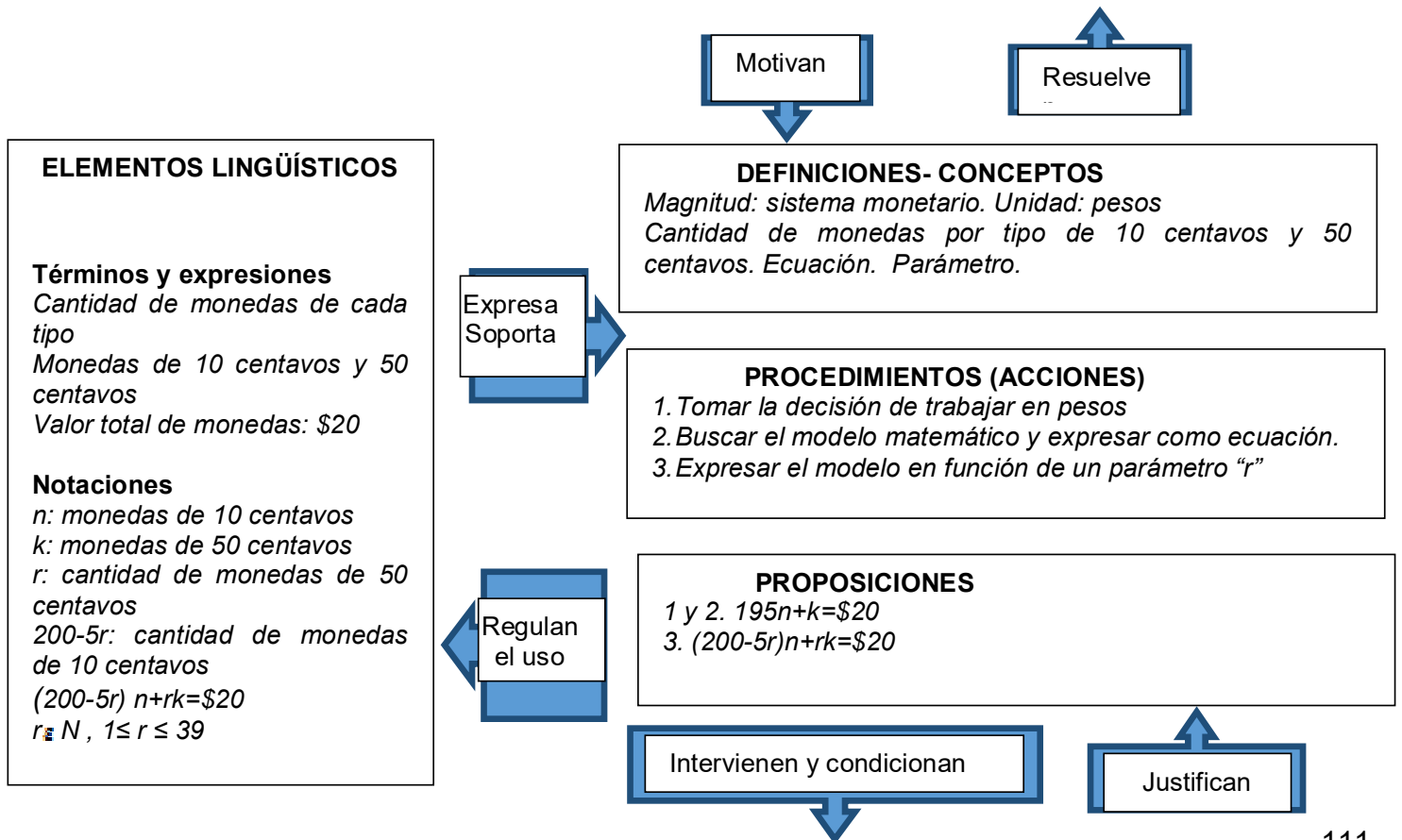




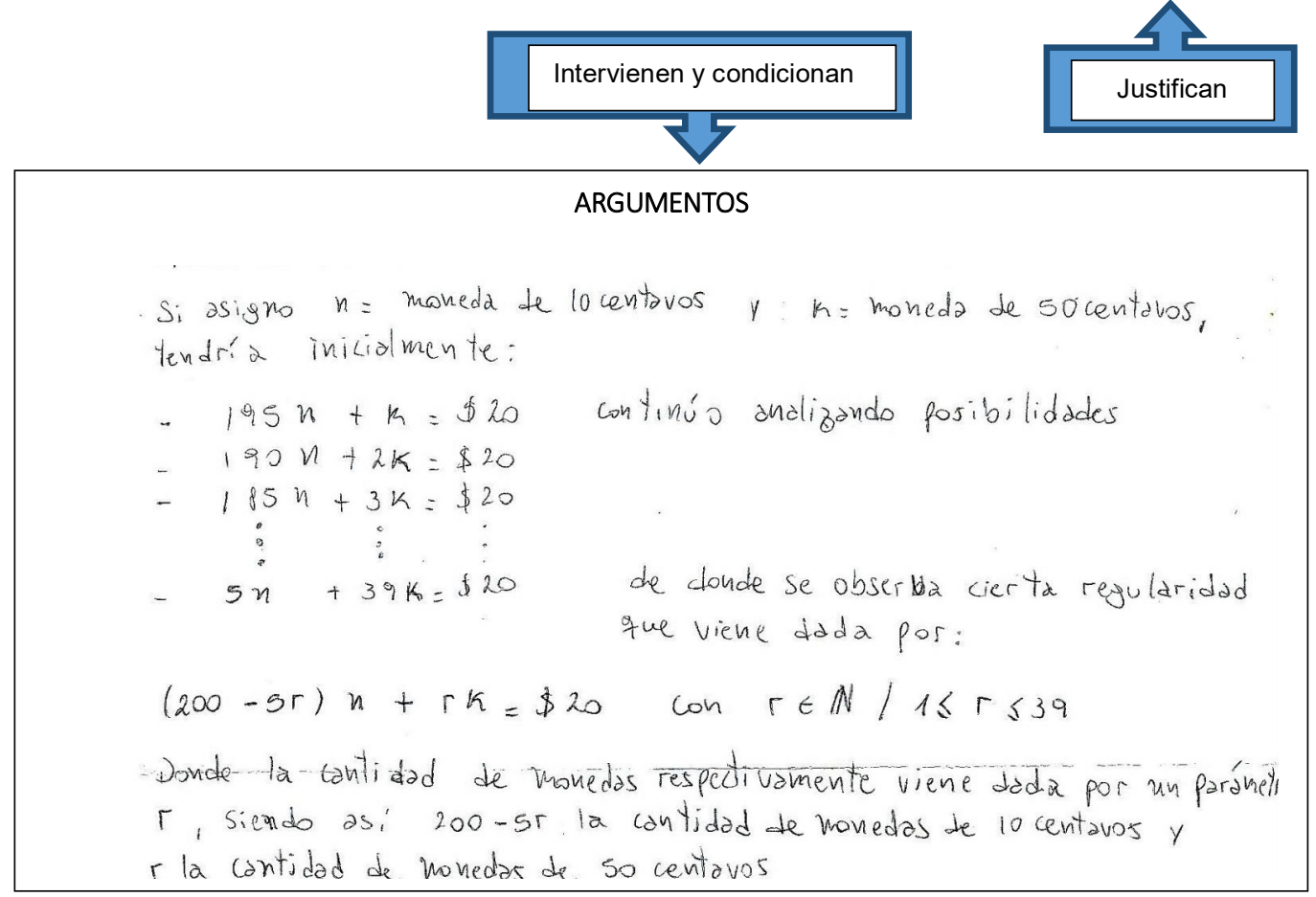

Fig.2 configuración cognitiva alumno 1

\subsubsection{Algunas consideraciones sobre la configuración del alumno ${ }^{1}$ (Fig.2)}

El lenguaje que utiliza en sus razonamientos y justificaciones es el natural y simbólico. El valor de las monedas de 10 y 50 centavos las representa a través de las letras ( $n$ y k), sin embargo, las mismas no son variables, son valores fijos. En su procedimiento presenta una secuencia de valores donde relaciona las cantidades de monedas de cada tipo de tal manera que la suma de 20 pesos, es decir va obteniendo valores particulares y probando su resultado, aunque en el registro no hay rastros de cómo obtiene las cantidades de monedas de cada tipo. Se observa que, a partir de éste razonamiento, reconoce una relación entre las cantidades de cada tipo de moneda, expresándola a través de una ecuación. Los objetos algebraicos intervinientes son los intensivos de grado 2, su proceso de generalización está expuesto como una regla $(200-5 r) n+r k=\$ 20$, que genera un conjunto finito de elementos expresados a través de un intervalo $(r \varepsilon N, 1 \leq r \leq 39)$.

\footnotetext{
1 Se tomó la idea del "formato propuesto" (como diagramas), de la tesis doctoral de Rocha Silva Gusmao (2006), para analizar los razonamientos y niveles de algebrización de los estudiantes
} 


\subsection{Configuración Cognitiva del alumno 2}

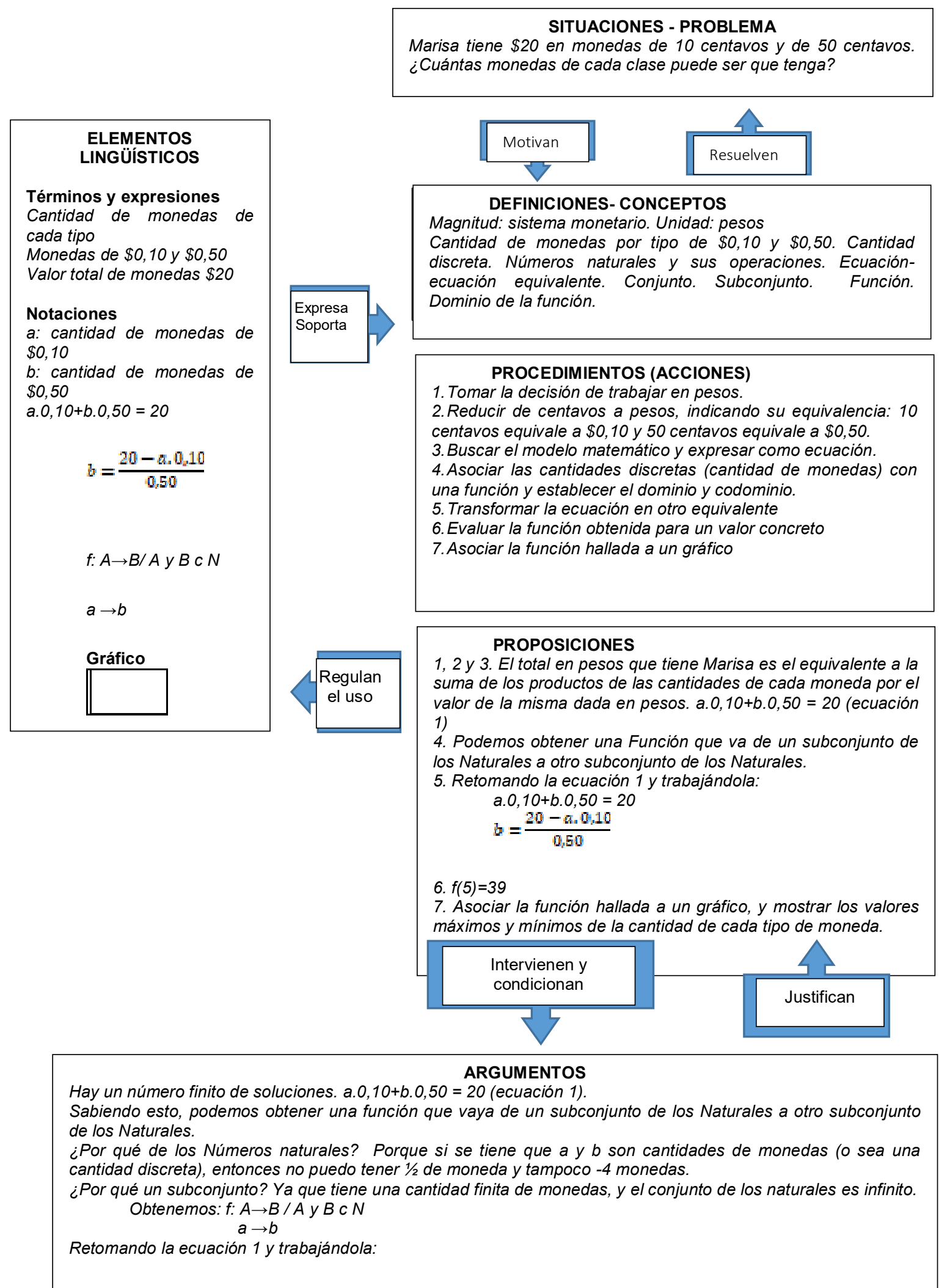




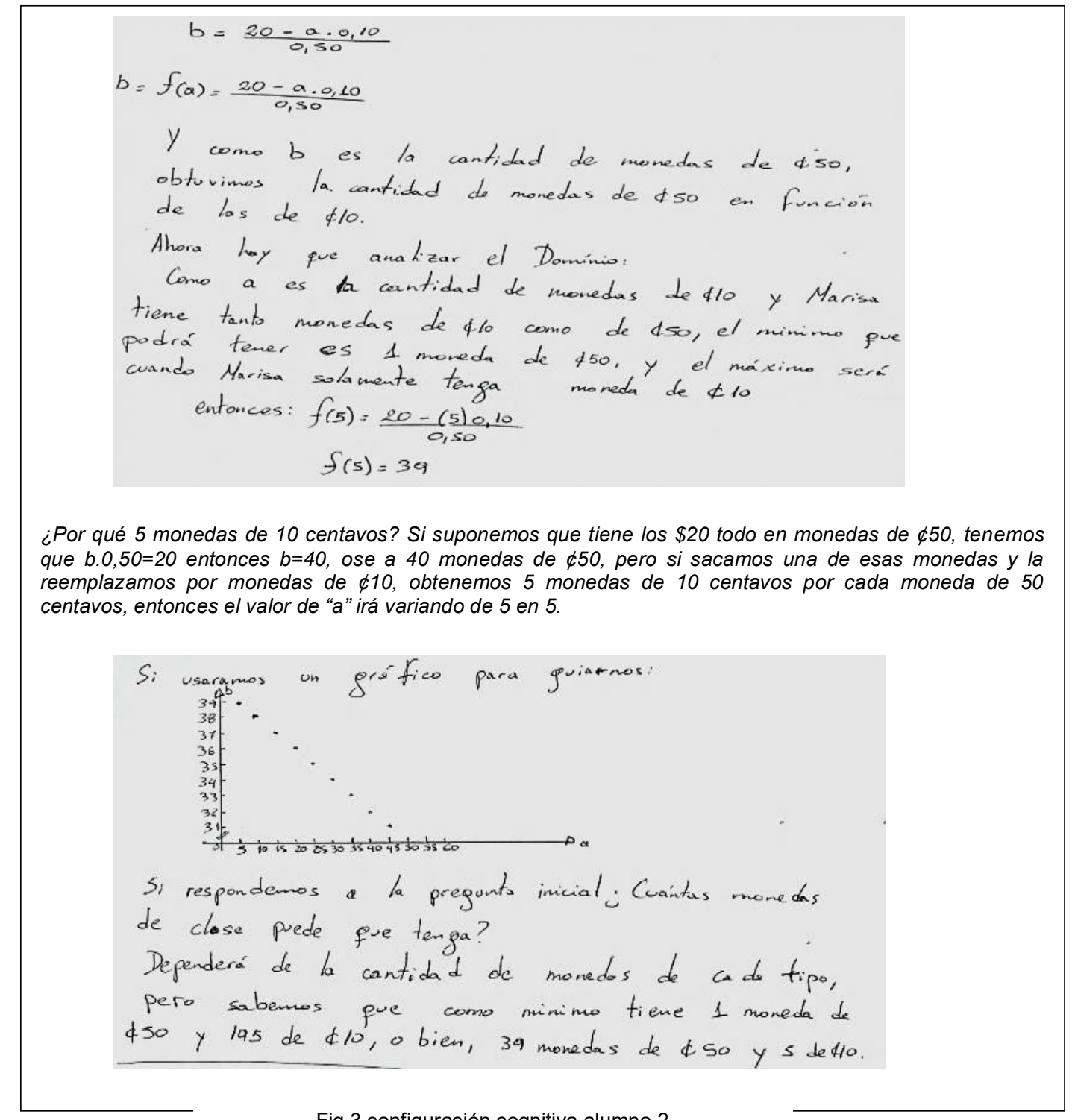

Fig. 3 configuración cognitiva alumno 2

\subsubsection{Algunas consideraciones sobre la configuración del alumno 2 (Fig.3)}

El lenguaje utilizado en sus razonamientos y justificaciones son: simbólico, natural, y gráfico. Los objetos algebraicos intervinientes son los intensivos de grado 2, ya que su proceso de generalización está mostrado como una regla, que genera un conjunto finito de elementos $(a .0,10+b .0,50=20)$ (ecuación 1). Intervienen incógnitas y ecuaciones (de la forma $A x+B y=C$ ), representadas de manera simbólica- literal. Opera y transforma analítica y sintácticamente. A partir de la ecuación obtenida (ecuación 1) la relaciona con una función. Los objetos intervinientes son variables y funciones particulares, donde se observa su proceso dual: la particularización donde obtiene objetos extensivos $(f(5)=39)$.

Utiliza una representación gráfica como guía de análisis de las soluciones, marcando algunos puntos, donde si bien, reconoce las variaciones de cada variable, las escalas que considera en los distintos ejes presenta problema, el corte que efectúa en uno de ellos (partiendo de 31), no le permite identificar todas las soluciones. 


\subsection{Configuración Cognitiva del alumno 3}

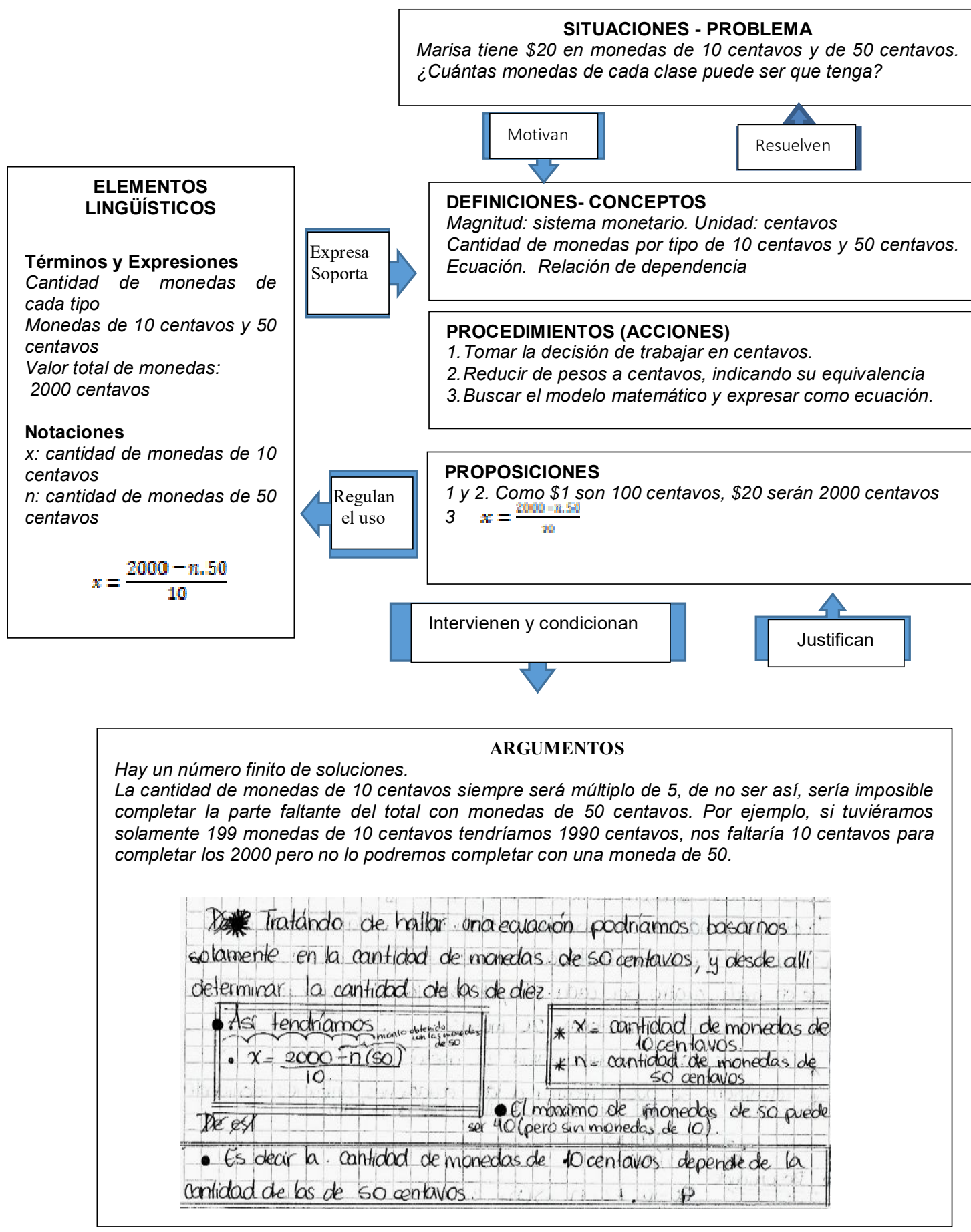

Fig.4 configuración cognitiva alumno 3

\subsubsection{Algunas consideraciones sobre la configuración del alumno 3 (Fig. 4)}

El lenguaje utilizado en sus razonamientos y justificaciones son: simbólico y natural, establece la conversión de pesos a centavos, reconociendo que $\$ 1$ son 100 centavos y $\$ 20,2000$ centavos. 
Distingue una relación de dependencia entre la cantidad de monedas de cada tipo cuando expresa: "la cantidad de monedas de 10 centavos depende de la cantidad de las de 50 centavos". Su proceso de generalización está presentado como una regla, no se evidencia la deducción de la misma. No se observan transformaciones en la forma simbólica de las expresiones conservando la equivalencia. Su generalización es de tipo mixta, contextual y simbólica.

\section{Algunas cuestiones observadas en las resoluciones de los problemas de los estudiantes}

De los 60 alumnos analizados, ninguno pudo identificar las 41 soluciones. Algunos se conformaron con "mostrar" una o a lo sumo 2 soluciones.

Otros plantearon las soluciones como intervalos, dónde expresaron entre qué valores podían tomar las monedas de cada tipo.

Por ejemplo: un alumno expresó: "la cantidad de monedas de 10 centavos va a estar entre 0 y 200 y las monedas de 50 centavos varían entre 0 y 40". No alcanzó a reconocer que las monedas de 10 no podían tomar todos los valores pertenecientes al intervalo considerado, a través de la ecuación: $10 x+50 y=2000$, dio un sólo valor a $x$ y obtuvo su correspondiente valor de y. En éste planteo, no se evidencia el reconocimiento de la cantidad total de soluciones, supone sólo la existencia de "varias", no tiene la certeza de cuántas.

Un solo alumno (alumno 4, Fig. 5), llegó a identificar de las 41 soluciones, 39 de ellas, utilizando un procedimiento no formal, con un lenguaje verbal-numérico. Sus argumentaciones estuvieron basadas en tipo de moneda, sin rastros del procedimiento o razonamiento utilizado, suponemos que lo hizo por tanteo. No consideró la posibilidad de tener una cierta cantidad de monedas de un sólo tipo.

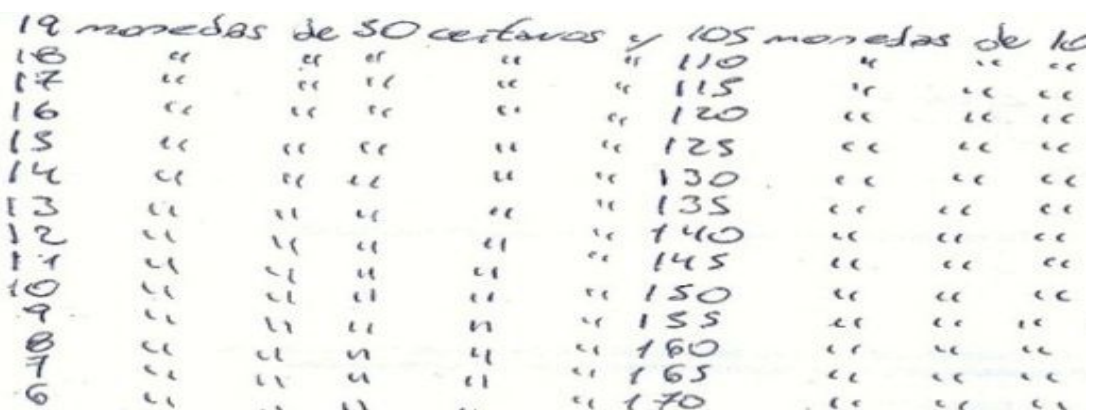

Fig. 5 razonamiento alumno 4

De los 60 alumnos analizados, ninguno logró considerar que podía existir la posibilidad de tener una cierta cantidad de un sólo tipo de monedas. El hecho de que, en el enunciado presentado, hablara de monedas de dos tipos, suponemos

Del total de alumnos analizados, ninguno logró considerar que podía existir la posibilidad de tener una cierta cantidad de un sólo tipo de monedas. El hecho de que, en el enunciado presentado,

De los alumnos analizados, ninguno logró considerar que podía existir la posibilidad de tener una cierta cantidad de un sólo tipo de monedas. El hecho de que, en el enunciado presentado, hablara de monedas de dos tipos, suponemos tal vez, los llevó a pensar que la solución estaba dada por la cantidad de monedas de cada una. Por ejemplo: El alumno 3, si bien, en un momento dado de su argumentación menciona la posibilidad de 0 monedas de 10 centavos, observamos que sólo lo declara con el objeto de deducir la regularidad de las monedas de 10 centavos (variar de 5 en 5). Podemos corroborar esto, porque observando el gráfico que ha efectuado y teniendo la posibilidad de considerar el punto $(0,40)$, no lo ha marcado.

Cabe aclarar que, de los estudiantes analizados, el estudiante 1, fue el único que en sus argumentaciones mencionó la palabra "parámetro". Sin embargo, si tenemos en cuenta las apreciaciones de Godino, et al. (2015), donde manifiestan que: "el uso de parámetros y su tratamiento puede ser criterio para delimitar niveles superiores de algebrización, ya que está ligado a familias de ecuaciones y funciones", esto no se observa en las argumentaciones de éste alumno. Es decir, el dar los valores en el intervalo ( $r \varepsilon N, 1 \leq r \leq 39)$ no obtiene una función, sino puntos que satisfacen la expresión encontrada.

Dos alumnos expresaron el conjunto solución por comprensión.

Por ejemplo, el alumno 5 (Fig.6), expresó en forma de pares ordenados, dónde ambas componentes son números naturales. Identifica las variables $\mathrm{x}$ e y e incorpora una nueva variable "t", que no es necesaria considerarla. En la expresión general como pares ordenados, no establece correctamente las condiciones de los valores del dominio, en lenguaje verbal expresa que las $\mathrm{x}$ 
consideradas deben ser números naturales de tal manera que "y" también lo deba ser. Además, su expresión simbólica para el conjunto solución no es correcta. La forma de considerar "t" perteneciente al conjunto de los naturales, no permite: a) encontrar los valores de "y" que sean números naturales, ya que sólo se podrá hallar para los múltiplos de 5 comprendidos entre 0 y 40 . b) hallar todas las soluciones del conjunto, lo conduce a que deba "encontrar" las soluciones "por tanteo".

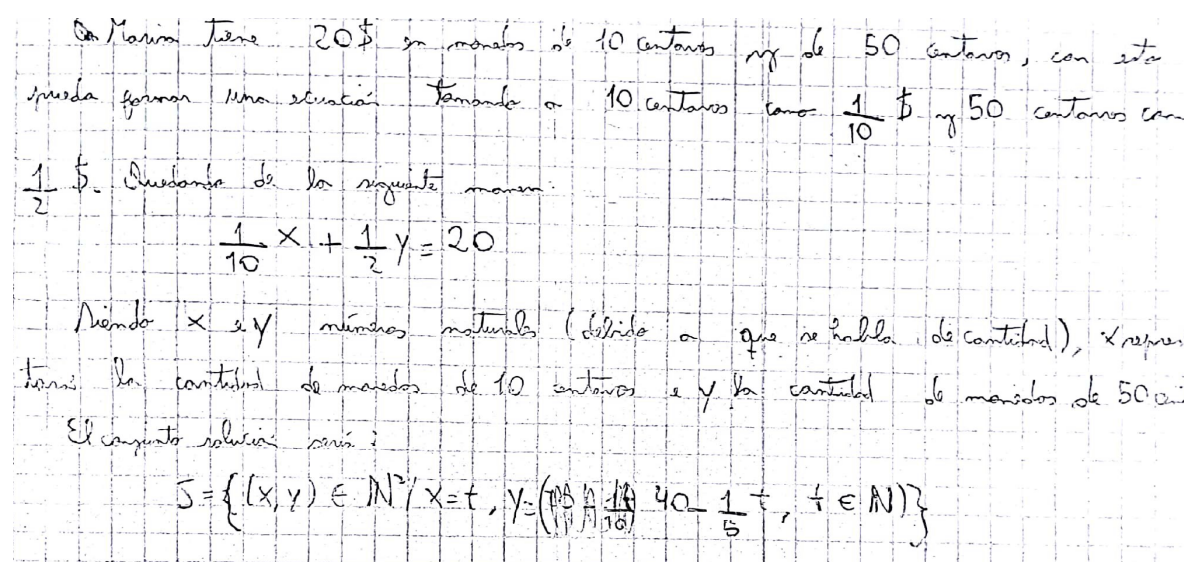

Fig.6 razonamiento alumno 5

Para la evaluación de la práctica matemática de los estudiantes analizados, fue importante a través de la situación- problema, como origen, observar los lenguajes utilizados por los mismos, tanto verbales, gráficos, etc., ya que fueron parte ostensiva de una serie de conceptos, proposiciones y procedimientos que intervinieron en la elaboración de sus argumentos y que nos sirvieron para analizar y decidir si las acciones y prácticas realizadas fueron satisfactorias o no, qué configuración cognitiva presentaron y sus niveles de algebrización.

La práctica matemática del alumno 1 con respecto a los objetos algebraicos mostrados, en donde intervienen ecuaciones y variables, son los intensivos de grado 2 . Reconoce un proceso de generalización expuesto como una regla, la cual genera un conjunto solución a través de un intervalo. Su nivel de algebrización es 2.

La práctica matemática del alumno 2, con respecto a los objetos algebraicos mostrados, reconoce la relación entre las variables, como así también identifica la ecuación y la asocia a una función, sin embargo, no logra evidenciar explícitamente todo el conjunto solución. De las 41 de ellas, sólo menciona algunas, no admitiendo como solución una cierta cantidad de un sólo tipo de moneda. Las argumentaciones utilizadas en sus justificaciones corresponden a un nivel de algebrización 3.

La práctica matemática del alumno 3 en relación a los objetos algebraicos mostrados, intervienen incógnitas y variables. Establece una equivalencia en cuanto a unidades de medidas (de pesos a centavos) relaciona las cantidades de las monedas de cada tipo. Su proceso de generalización está presentado como una regla. Reconoce explícitamente una solución. Establece una dependencia entre variables y detrás de ésta idea queda implícita la existencia de más soluciones, aunque no específica cuántas. Por todo lo expuesto, el nivel de algebrización es 2.

Con respecto al alumno 4 que logró identificar 39 de las 41 soluciones, ha utilizado en sus argumentaciones casos particulares, los objetos intervinientes fueron intensivos de primer grado. Estos procesos de particularización no son considerados propios del razonamiento algebraico, es decir hay una ausencia del mismo, por ello sostenemos que su nivel de algebrización es 0 .

La práctica matemática del alumno 5, con respecto a los objetos algebraicos involucrados, muestra la equivalencia de unidad monetaria, identifica incógnitas, establece las relaciones entre variables, ecuaciones, conjunto solución. En su proceso de generalización, exhibe una ecuación y el conjunto solución (por comprensión). Presenta un manejo no adecuado en el uso de la simbología. Su nivel de algebrización es 3 .

Teniendo en cuenta el número de soluciones que se debía responder: 41, en general los alumnos analizados, sólo se limitaron a presentar algunas de ellas y validarlas. En tal sentido y coincidiendo con las ideas de Sadovsky y Sessa (2004), que lograr una generalización, no da información suficiente para encontrar la cantidad de soluciones. Si bien los análisis realizados por estas investigadoras correspondieron a alumnos de nivel primario, es preocupante observar que continúa persistiendo las mismas dificultades en alumnos de niveles superiores. 
Pensamos que realizar éste tipo de estudio y profundización en todo lo referente a la problemática algebraica, contribuye a tomar conciencia de las fisuras y discontinuidades que existen en el aprendizaje de ésta área.

Por último, se pone de manifiesto, cómo la actividad matemática puesta en juego en una determinada tarea puede estar situada en un plano aritmético y cómo la manifestación de determinados rasgos algebraicos la podría situar en un nivel diferente de algebrización. Es decir, frente a una misma situación problema, se encuentran diferentes configuraciones cognitivas y niveles de algebrización en los estudiantes del profesorado.

\section{Referencias bibliográficas}

Aké, L. P.; Godino, J. D. y Castro, W. F. (2015). Distinción del pensamiento algebraico del aritmético en actividades matemáticas escolares. Ruiz, A. (Presidente). XIV Conferencia Interamericana de Educación matemática. Tuxtla Gutiérrez, Chiapas, México.

Godino, J. D. y Batanero, C. (1994). Significado institucional y personal de los objetos matemáticos. En: Recherche en Didactique des Mathématiques, Grenoble, 14(3), p. 325-355.

Godino, J. D.: Batanero, C. y Font, V. (2009). Un enfoque ontosemiótico del conocimiento y la instrucción matemática. Versión ampliada y revisada artículo, J. D.; C. Godino, Batanero y V. Font, (2007). En: The ontosemiotic approach to research in mathematics education.ZDM. The International Journal on Mathematics Education, 39 (1-2), p. 127-135.

Godino, J. D.; Aké, L. P.; Gonzato, M. y Wilhelmi, M. R. (2014). Niveles de algebrización de la actividad matemática escolar. Implicaciones para la formación de maestros. En: Enseñanza de las Ciencias, 32(1), p. 199-219.

Godino, J. D., et. al. (2015). Diseño de un cuestionario para evaluar conocimientos didáctico matemáticos sobre razonamiento algebraico elemental. En: Enseñanza de las Ciencias, 33(1), p. 127-150.

Godino, J. D., et. al. (2015). Niveles de algebrización de las prácticas matemáticas escolares. Articulación de las perspectivas ontosemiótica y antropológica. Trabajo realizado en el marco de los proyectos de investigación, EDU2012-31869 y EDU2013-41141-P, Ministerio de Economía y Competitividad (MINECO).

Rocha Silva Gusmão, T. (2006). Los procesos metacognitivos en la comprensión de las prácticas de los estudiantes cuando resuelven problemas matemáticos: una perspectiva ontosemiótica (Tesis doctoral). Universidad de Santiago de Compostela, España.

Sadovsky, P. (2003). Condiciones didácticas para un espacio de articulación entre prácticas aritméticas y prácticas algebraicas. (Tesis doctoral) Universidad de Buenos Aires, Argentina.

Sadovsky, P y Sessa, C (2004). la interacción adidáctica con los procedimientos de los otros en la transición aritmética algebra: un milieu para la emergencia de nuevas preguntas. En: Revista Educational Studies in Mathematics (2005). Vol.59, pp. 85-112. 